\title{
Une histoire culturelle du missionnaire : Julien Moulin, du diocèse de Rennes au Nord-Ouest canadien, 1830-1878
}

Tangi Villerbu

\section{(2) OpenEdition \\ Journals}

Édition électronique

URL : http://journals.openedition.org/abpo/418

DOI : $10.4000 / a b p o .418$

ISBN : 978-2-7535-1507-9

ISSN : 2108-6443

\section{Éditeur}

Presses universitaires de Rennes

Édition imprimée

Date de publication : 30 septembre 2007

Pagination : 7-34

ISBN : 978-2-7535-0554-4

ISSN : 0399-0826

\section{Référence électronique}

Tangi Villerbu, « Une histoire culturelle du missionnaire : Julien Moulin, du diocèse de Rennes au NordOuest canadien, 1830-1878 », Annales de Bretagne et des Pays de l'Ouest [En ligne], 114-3 | 2007, mis en ligne le 30 septembre 2009, consulté le 20 avril 2019. URL : http://journals.openedition.org/abpo/418 ; DOI : 10.4000/abpo.418 


\title{
Une histoire culturelle du missionnaire : Julien Moulin, du diocèse de Rennes au Nord-Ouest canadien, 1830-1878
}

\author{
Tangi VILLERBU \\ Docteur en histoire, chercheur associé au Centre d'études nord-américaines, \\ CENA-MASCIPO, EHESS, UMR CNRS 8168 \\ lycée Émile-Littré - Avranches
}

Julien Moulin fut un missionnaire breton, né en 1830, arrivé dans le Nord-Ouest canadien en 1858, et comme ses confrères Oblats de Marie Immaculée, il eut en charge des populations indiennes et métisses jusqu'à sa mort, en 1920. Il pourrait disparaitre sous la statistique collective à plusieurs titres : comme fils d'artisan breton, comme séminariste français, comme missionnaire oblat, il ne se distingue $a$ priori en rien, et ne devrait être que noyé dans les masses des anonymes et des invisibles de l'histoire. Mais une particularité rend son cas exceptionnel : les archives de sa paroisse d'origine, La Gouesnière, non loin de Saint-Malo, conservent un ensemble de lettres qu'il a adressées à sa famille entre 1854 et 1878 et incitent à se pencher sur ce destin individuel, non pas uniquement pour lui-même mais pour ce qu'il peut apporter à une connaissance plus globale d'un ensemble de phénomènes.

Il ne s'agit pas ici de faire une biographie de Julien Moulin, mais une histoire culturelle du missionnaire, c'est-à-dire d'abord de la société et de la culture qui le produisent et le construisent, et de sa rencontre avec une autre société, une autre culture, qui le remettent en cause et dont il tente lui-même d'appréhender les ressorts. Cette histoire est évidemment aussi religieuse, car elle éclaire à la fois le catholicisme français et l'histoire de la mission. C'est d'une part une paroisse du diocèse de Rennes qui voit son fonctionnement éclairé pour le milieu du XIXe siècle, c'est d'autre part un renouveau de l'histoire de l'expansion catholique qui doit être à l'œuvre. Dans le premier cas, il s'agit de couvrir une période mal connue localement ${ }^{1}$

1. Le XIX ${ }^{\mathrm{e}}$ siècle n'est guère connu dans le détail que par LAGRÉE Michel, Mentalités, religion et histoire en Haute-Bretagne au XIX $x^{e}$ siècle. Le diocèse de Rennes 1815-1848, Paris, Klincksieck, 1977, 492 p. 
et de s'ouvrir à l'échelle paroissiale peu utilisée dans l'historiographie du XIX ${ }^{\mathrm{e}}$ siècle français alors qu'elle est celle du quotidien des fidèles ${ }^{2}$, dans le deuxième il s'agit d'aller au-delà des renouvellements du champ qui ont mené à une meilleure compréhension des populations cibles des missions et de leurs réactions à celles-ci, pour revenir sur la figure du missionnaire qui demeure trop souvent objet hagiographique, à moins qu'elle ne soit rejetée dans l'ombre de ses ouailles ou de l'étude collective. En somme il s'agit d'une histoire transatlantique, pour observer un migrant d'un type particulier grandir et naître à sa vocation sacerdotale puis missionnaire en Bretagne avant de partir pour ce qui lui est inconnu, exercer sa profession dans l'actuelle Saskatchewan tout en demeurant un fils de La Gouesnière.

Ce passage par l'échelle individuelle semble indispensable en histoire religieuse. Claude Langlois théorise lui-même son passage lent d'une histoire globale et monumentale des congrégations féminines à une approche intellectuelle de Thérèse de Lisieux en arguant de l'impossible approche de la foi et du choix de la vie religieuse sans choisir cette voie ${ }^{3}$. Raymond Huel constate de même qu'une histoire des Oblats dans le Nord-Ouest canadien ne saurait se concevoir sans s'attacher aux individus tant cette histoire est faite d'" aspirations et de caractères personnels qu'il est parfois difficile de concilier avec les ordres et les vœux religieux ${ }^{4}$ ". Le cas de Julien Moulin permet justement d'envisager cette échelle fine, paroissiale et individuelle, de l'histoire de la mission, de la production du missionnaire à l'exercice quotidien de son métier.

\section{La famille Moulin, de la paroisse de La Gouesnière}

Il n'est pas envisageable de comprendre un missionnaire dans son exercice sur le terrain sans s'interroger sur l'itinéraire qui l'y a mené, sur le terreau qui l'a vu naître. Julien Moulin, au moins jusqu'en 1878 - les archives paroissiales ne conservent aucune correspondance ultérieure - garde contact avec sa famille ou avec les prêtres de La Gouesnière. C'est pour lui d'une importance primordiale. La Gouesnière, dans l'histoire personnelle de Julien Moulin, c'est autant sa famille que sa paroisse, les deux étant liées l'une à l'autre.

\section{Une famille de petits notables de village}

La famille Moulin est, entre 1830 et 1858 - entre la naissance de Julien Moulin et son départ pour le Canada -, une des familles qui comptent à La

2. Restif, Bruno, La Révolution des paroisses : Culture paroissiale et Réforme catholique en Haute-Bretagne aux XVI et XVIT siècles, Rennes, PUR, 2006, 415 p.

3. LANGLOIS, Claude, Les Congrégations féminines à supérieure générale au XIXe siècle, Paris, Fayard, 1984, 776 p. et Le Désir de sacerdoce chez Thérèse de Lisieux, Paris, Salvator, 2002, 230 p.

4. Huel, Raymond J. A., Proclaiming the Gospel to the Indians and the Métis, Edmonton, University of Alberta Press, 1996, p. XXI. 
Gouesnière, par son enracinement et par son statut social. Il faut pour le comprendre remonter aux quinze dernières années du XVIII ${ }^{\mathrm{e}}$ siècle, lorsque deux frères, Philippe et Michel Moulin, donnent naissance à onze enfants, quatre pour le premier, sept pour le second. Philippe est charron, Michel est charpentier, ils sont tous deux alphabétisés et membres des cercles de notables du village avant et pendant la Révolution. Ils le demeurent après, et leurs enfants les suivent dans cette voie. Philippe a quatre fils : Allain, laboureur, qui épouse Modeste Fouré, d'une famille de notables locaux également; Joseph; Michel, laboureur également, dont le mariage avec Perrine Solier lie la famille Moulin avec celle qui donne les maires de La Gouesnière dans les premières décennies du XIX ${ }^{\mathrm{e}}$ siècle; Phillippe, qui reprend le métier paternel de charron. Michel, lui, a six fils : c'est l'aîné Guillaume, né en 1788, qui devient charpentier, tandis que Michel fils - marié à Marie Fouré -, Joseph et Jean se font laboureurs, ce qui suppose comme pour leur cousin les moyens de s'installer, et que Julien est comme son oncle charron; il a aussi une fille, Jeanne, qui est donnée en mariage à Guillaume Lochet, que le sous-préfet de Saint-Malo propose comme conseiller municipal de Bonnaban (commune rattachée à La Gouesnière en 1829) en 1827 aux côtés de ses beaux-frères Michel Moulin fils et Guillaume Moulin ${ }^{5}$.

Sous la Restauration et la monarchie de Juillet, au temps du suffrage censitaire, les Moulin sont impliqués dans la vie politique de la commune, comme électeurs et comme élus. Ceci suppose d'abord une fortune suffisante pour être présent sur les listes d'électeurs qui ne comportent qu'environ $10 \%$ des habitants de la commune (90 sur 900 en moyenne) et ensuite à la fois la volonté de s'imposer sur la scène locale et l'entregent suffisant pour le faire. Les listes de nomination aux conseils municipaux de la Restauration subsistent, pour les deux communes de Bonnaban et La Gouesnière. Les Solier (Michel est maire dès 1808), les Fouré et les Lochet, les trois familles au sein desquelles se marient les Moulin, y abondent durant toute la période. À Bonnaban Michel fils et Guillaume apparaissent en 1827, à La Gouesnière Allain est conseiller en $1830^{6}$. La monarchie de Juillet a laissé localement de vastes archives. Durant les dix-huit années que dure le régime, les Moulin sont entre 4 et 6 parmi les électeurs : Michel père, qui l'est encore en 1846 à 98 ans, alors que son frère est décédé en 1828 à 78 ans, et la génération suivante, sans que tous y parviennent ce qui dénote des réussites bien différenciées au sein de la famille : Michel fils, Philippe fils, Joseph et surtout Allain s'y distinguent. Ce dernier occupe avec une singulière constance un rang entre le $15^{\mathrm{e}}$ et le $18^{\mathrm{e}}$ dans la liste des citoyens imposables de la commune, sachant que parmi les premiers on trouve nombre de propriétaires absentéistes dont l'implication dans la vie politique locale est nulle. Avec Michel fils,

5. Arch. dép. d'Ille-et-Vilaine, 2 M 53, Commune de Bonnaban, nomination des conseils municipaux sous la Restauration.

6. Idem et 2 M 55, Commune de La Gouesnière, nomination des conseils municipaux sous la Restauration. 
il est élu au conseil municipal en 1831, et les deux cousins sont réélus en 1837 comme en 1843. 1848 apporte des changements, bien sûr, mais dans la continuité. Le suffrage universel n'entame pas les carrières de tous les notables locaux : si les Solier disparaissent, ce n'est pas le cas des Fouré, pas plus que celui des Moulin puisque les deux mêmes sont toujours élus, sous la République comme dans les premières années du Second Empire. Mais Allain Moulin, malgré sa richesse, symbolisée par le montant de ses impôts comme par ses quatre domestiques recensés en $1851^{7}$, bien qu'arrivé en tête des suffrages à égalité de voix avec Henri de Kergariou, est battu par ce dernier pour le poste de maire : Kergariou, un aristocrate légitimiste qui avait acquis en 1841 le château de Bonnaban, obtient 11 voix, contre 1 à Allain Moulin ${ }^{8}$.

Julien Moulin fils, qui naît en 1830, vit donc ses années bretonnes au sein des cercles dominants du village, tant d'un point de vue économique que politique. Mais la place de son propre père, Julien Moulin également, semble quelque peu en deçà de celle de son frère Michel ou de son cousin Allain, comme de celle de son père et de son oncle. Julien père naît le 12 brumaire an V (1797), et son père, Michel Moulin, lui donne comme témoin Michel Solier, le futur maire de la commune, charpentier comme lui. C'est pourtant Guillaume, le frère aîné, qui reprend la profession paternelle, Julien devenant charron, comme son oncle Philippe et son cousin du même nom, ce qui explique peut-être qu'il soit le seul des enfants de Michel à s'installer, avant $1851^{9}$, au bourg de La Gouesnière, comme ses deux parents et sa nièce Marie du fait de son mariage avec l'instituteur, Pierre Lerède. Il est donc physiquement à l'écart de ses frères et de son père.

En terme de revenus, il se distingue également : il n'est sur les listes des électeurs qu'à deux occasions, en 1839 et en 1843. En 1839, il occupe la quatre-vingt-deuxième place sur quatre-vingt-dix, avec 27,26 francs de contributions alors que la même année son cousin Allain en paye 137,32, son autre cousin Philippe 48,09 et son frère Michel 47,27. En 1843, il est quatre-vingt-unième, pour payer une somme similaire. Il n'est donc pas celui des Moulin qui a réussit le mieux, mais dans le même temps ses frères Guillaume, Joseph et Jean sont encore moins bien lotis et sa situation laisserait à penser à une réussite naissante autour de 1840. Mais si ce fut le cas elle fut fugace car dès 1846 il perd son statut d'électeur ${ }^{10}$.

Julien père et sa famille sont donc dans un entre-deux social. Le fait est confirmé par la nature de son mariage. Alors que la norme était dans

7. Arch. dép. d'Ille-et-Vilaine, 6 M 231, commune de La Gouesnière, recensement de 1851.

8. Arch. dép. d'Ille-et-Vilaine, E Dépot adm La Gouesnière 2, Délibérations du conseil municipal 1839-1892.

9. Le recensement, à partir de cette date, l'indique comme habitant le bourg, alors qu'il est dit résidant Laval, un écart, lors de la naissance de ses enfants vingt ans auparavant. Arch. dép. d'Ille-et-Vilaine, 6 M 231 (voir ci-dessus) et 5 Mi 129, registres d'état-civil de La Gouesnière.

10. Arch. dép. d'Ille-et-Vilaine, 3 M 127 (déjà cité) et E Dépot adm La Gouesnière 6-8, commune de La Gouesnière, liste des électeurs communaux. 
la famille de se lier avec les autres familles dominantes de la commune, Julien épouse une " étrangère ", Julienne Loisel, qui n'est originaire ni de La Gouesnière ni des communes voisines ${ }^{11}$. Et il lui fut difficile de créer luimême des liens supplémentaires, même s'il l'a souhaité : il n'eut que deux enfants, dont les destins lui ont interdit de rêver à ce type de pratique. Julien fils, qui naît le 27 juillet 1830, est celui qui part au séminaire puis en mission au Canada; Marie, née le 15 décembre 1831, réside chez ses parents sans se marier jusqu'à sa mort précoce, à l'âge de 32 ans, le 14 juillet 1863, dans la maison familiale. Il est un domaine où Julien père est une fois de plus en deçà de son frère Michel tout en lui étant associé de près : la gestion de la fabrique.

Il s'agit d'un secteur d'où est absent Allain Moulin, ce qui fournit peutêtre un élément d'explication à sa défaite de 1848 face à Kergariou : le peu d'implication dans les affaires paroissiales alors que la fabrique, telle qu'elle est rétablie suite au concordat de 1802, se doit de rassembler les notables dans la gestion temporelle du religieux, signale peut-être un éloignement de la foi. Ce n'est pas le cas de nombreux autres membres de la famille Moulin. Si l'on ne tient compte que des archives de la fabrique - mais il en existe peu d'autres qui donnent accès au sentiment religieux des paroissiens individuellement -, les Moulin seraient des croyants exemplaires. Dès 1811 en effet le maire, alors Michel Solier, propose comme membre du conseil de fabrique Philippe Moulin - sans doute le père mais ce n'est pas précisé -, proposition acceptée par le sous-préfet ${ }^{12}$. À partir de 1824 , et jusqu'à la fin du XIX ${ }^{\mathrm{e}}$ siècle, les registres des délibérations du conseil sont conservés. L'organisation de la fabrique fut longue à se dessiner, les structures paroissiales longues à remettre en place. Le 10 avril 1825 est inauguré un nouveau conseil : en fait partie Michel Moulin père, nommé par le préfet. Il siège aux côtés, entre autres, de Michel Solier et de Valentin Fouré, à qui sa famille est liée par des mariages. Dans les années suivantes, lui-même et son fils Michel sont bénéficiaires des travaux réclamés et payés par la fabrique (Michel père avait déjà obtenu par adjudication la restauration du presbytère en $1807^{13}$ ), tandis que Philippe Moulin en 1832 et Joseph en 1839 font leur entrée au sein de la même assemblée, tout comme Julien Moulin père en 1841. Durant quelques mois, en 1837, Michel père est même trésorier de la fabrique, mais celle-ci connaît très certainement des problèmes de fonctionnement : les trésoriers se succèdent très rapidement et les renouvellements se font de manière de plus en plus désorganisée ${ }^{14}$.

En 1845 un nouveau conseil doit être nommé, conjointement par l'évêque de Rennes, Godefroy Saint-Marc, et par le sous-préfet de Saint-Malo.

11. À une date inconnue puisque une partie des registres de l'ancienne commune de Bonnaban a disparu en 1944 à Saint-Malo.

12. Arch. dép. d'Ille-et-Vilaine, 1 V 742.

13. Arch. dép. d'llle-et-Vilaine, 2 O 123/11, papiers administratifs et financiers concernant l'église de La Gouesnière.

14. Arch. dép. d'Ille-et-Vilaine, 5 V 127/2, paroisse de La Gouesnière, registre des délibérations du conseil de fabrique, 1824-1845. 
On retrouve alors Michel Moulin, et surtout Philippe Moulin fils comme président et Julien Moulin père comme trésorier. La situation demeure longtemps inchangée : en 1856, alors que Julien Moulin fils est déjà au noviciat des Oblats à Notre-Dame-de-L'Osier dans l'Isère, son cousin Philippe perd la présidence au profit d'Henri de Kergariou mais demeure vice-président. Quant à Julien père, il ne cède sa place de trésorier qu'en 1868, à 71 ans, et encore est-ce Philippe Moulin, troisième du nom, qui lui succède ${ }^{15}$. Julien Moulin fils est donc l'aîné de l'inamovible trésorier de la fabrique, une fabrique qui aura eu à gérer parmi ses grandes affaires le testament de Michel Moulin, qui lègue en 1860 une rente annuelle de 175 francs destinée à payer cent messes basses ${ }^{16}$. Il n'y a guère de doute que le futur Oblat a été élevé dans une famille au sein de laquelle la foi demeurait vivace et pour laquelle il s'agissait non seulement de croire mais d'agir au quotidien. Reste à analyser quel pouvait être ce catholicisme à La Gouesnière dans ces années 1830-1850, puisque cette paroisse a produit un missionnaire.

\section{La production du missionnaire}

Au centre de la paroisse "règnent " le recteur et son vicaire. Julien Moulin, lorsqu'il sera au Canada, n'omettra jamais de signaler à ses parents de leur faire part de son respect et de leur donner de ses nouvelles, quand il ne leur écrira pas lui-même directement, comme à Auguste Feuillet, arrivé pourtant dans la paroisse en 1866, longtemps après le départ du missionnaire. C'est au contact de ces desservants que peut-être la vocation de Julien Moulin est apparue, c'est en tout cas à leur contact que la foi se nourrit. Les sources manquent pour étudier leur personnalité et leur rôle précis, leur profession au quotidien, comme elles manquent plus généralement pour une histoire paroissiale de la culture catholique - encore que La Gouesnière ne soit pas la plus mal lotie des paroisses de ce point de vue - mais il existe des bribes archivistiques permettant d'ouvrir une petite fenêtre sur la vie de certains de ces prêtres.

Julien Moulin a connu, entre sa naissance et la fin des années 1870 , quatre recteurs et trois vicaires, c'est-à-dire en fait six prêtres puisque l'un d'entre eux a occupé successivement les deux postes. Ils partagent tous des traits communs. François Hue, de 1820 à 1845, Adolphe Baudouin de 1845 à 1862, puis Gilbert Vromet de 1862 à 1866 et enfin Auguste Feuillet de 1866 à 1888 sont tous des enfants du pays, tous comme leurs vicaires, Adolphe Baudouin déjà, entre 1830 et 1845, Jean Duval, de 1845 à 1854 et Jean-François Chauchix entre 1854 et 1872. En effet Baudouin et Vromet sont natifs de Cancale, Duval de Saint-Coulomb, Chauchix de Plesder. Leur carrière est en fait des plus classique : après le séminaire, ils occupent un ou plus rarement plusieurs postes de vicaires dans leur diocèse d'origine

15. Arch. dép. d'Ille-et-Vilaine, 5 V 127/1, paroisse de La Gouesnière, registre des délibérations du conseil de fabrique, depuis 1845 .

16. Arch. dép. d'Ille-et-Vilaine, 5 V 127/6 et Arch. dép. d'Ille-et-Vilaine, 4 V 135. 
avant de devenir eux-mêmes recteurs, dans une autre paroisse ou plus rarement la même - c'est le cas de Baudouin, qui totalise 32 ans de présence à La Gouesnière - à 44 ans pour Baudouin ou Vromet, 48 pour Hue, avec la tentation bien souvent de se rapprocher de sa commune de naissance ${ }^{17}$.

Ces prêtres sont d'autant plus caractéristiques qu'ils se distinguent peu par des qualités exceptionnelles : La Gouesnière a été desservie par un personnel moyen durant des décennies, comme sans doute la grande majorité des paroisses du diocèse. $\mathrm{M}^{\mathrm{gr}}$ de Lesquen, peu brillant par ailleurs, ancien émigré, tient comme il se doit un registre du personnel. François Hue y est indiqué comme ayant des capacités, "bon jugement, bon ton ${ }^{18}$ ". Un recteur honnête, en somme, qui agit comme on l'attend. Jean-François Chauchix suscite plus de doutes. Godefroy Brossays Saint-Marc, successeur de Lesquen, plus ambitieux, commence un nouveau registre en 1851 et le vicaire de La Gouesnière y est décrit de la sorte : "Capacité médiocre. Bon ensemble. Un peu lent et timide. Santé faible ${ }^{19}$. " Les visites annuelles, en juillet de chaque année entre 1855 et 1858 reprennent le jugement d'ensemble ${ }^{20}$. Vromet, lui, suscite ce commentaire : "Capacité ordinaire. Travail ordinaire. Piété. Bon caractère - un peu causeur et curieux ${ }^{21}$. " Par contre Baudouin et Duval, ceux que Julien Moulin a sans doute le plus fréquenté dans sa jeunesse, ne sont l'objet d'aucun commentaire alors qu'existent des rubriques à leur nom, soit qu'il y ait eu absence de jugement, soit que celui-ci n'ait jamais été porté, pour une raison ou pour une autre.

La présence de ces prêtres aux origines locales, à la carrière très normée, et au caractère peu marqué, a sans doute eu une influence sur la famille de Julien Moulin : sur lui-même sans doute car ce sont eux qui lui ont enseigné la religion, sur ses parents aussi car lorsque Julien entre au séminaire en 1852, c'est sans annoncer de vocation missionnaire et donc en laissant présager une carrière de ce type, un enracinement de longue durée dans le pays malouin et l'apparition d'un notable de plus dans la famille. Les attentes devront alors être réorientées.

La paroisse de La Gouesnière est aussi transparente, d'une certaine manière, que ses desservants. Il y existe bien deux confréries, les réponses de François Hue à l'enquête lancée par M ${ }^{\text {gr }}$ de Lesquen en 1835 en attestent ${ }^{22}$ : le $1^{\text {er }}$ août 1829 ont été érigées les confréries du rosaire et de Marie

17. Arch. dép. d'Ille-et-Vilaine, 5 V 127/1, livre de paroisse de La Gouesnière. Arch. dép. d'Ille-et-Vilaine, 6 V 88, registre du personnel du clergé du diocèse de Rennes sous l'épiscopat de Brossays Saint-Marc. Arch. dép. d'Ille-et-Vilaine, 6 V 94, registre du personnel du clergé du diocèse de Rennes sous l'épiscopat de Lesquen. 6 V 98, registre des mutations du personnel du diocèse de Rennes, s. d.

18. Arch. dép. d'Ille-et-Vilaine, 6 V 94 (cité plus haut).

19. Arch. dép. d'Ille-et-Vilaine, 6 V 88 (cité plus haut).

20. Arch. dép. d'Ille-et-Vilaine, 6 V 94, livret d'examen des vicaires du diocèse de Rennes, années 1850.

21. Arch. dép. d'Ille-et-Vilaine, 6 V 94 (cité plus haut).

22. Arch. dép. d'Ille-et-Vilaine, 6 V 145, enquête de $\mathrm{M}^{\mathrm{gr}}$ de Lesquen sur l'état des paroisses du diocèse de Rennes, 1835. 
(celle-ci n'étant que réactivée car elle date en fait de 1664), qui doivent aider le recteur à mettre en place sa pastorale simple, faite de « moralisme étroit et [de] crainte de la mort ${ }^{23}$ " mais dont on ne sait rien par ailleurs. Le dossier est plus complet sur une relique que possède la paroisse car en $1831 \mathrm{M}^{\mathrm{gr}}$ Lesquen avait ordonné à son sujet une enquête ${ }^{24}$. Il en était ressorti que la " parcelle de la vraie croix " connue à cette époque était bien celle qui existait sous l'Ancien Régime, et qui avait été confiée par le prêtre constitutionnel à un de ses paroissiens, Soligné, afin qu'il la dissimule. Le dit Soligné l'avait lors du retour du roi confié à Anne Solier, "qui était en charge du soin de l'église " et qui l'avait elle-même remise à son frère Michel Solier, en poste à la mairie sous l'Empire comme sous la Restauration. La relique doit désormais " être exposée à la vénération publique ", c'est-à-dire que la croix de cristal dans laquelle elle est enchâssée doit être "renfermée dans un cœur en argent, ayant une vitre sur une partie de sa surface". Quatre ans plus tard, Hue signale que la paroisse possède " seulement ${ }^{25}$ " la dite relique et il ne se développera apparemment aucune dévotion particulière autour d'elle. Ainsi en 1861, lors de l'enquête sur les paroisses du diocèse diligentée cette fois par Brossays Saint-Marc, Adolphe Baudouin ne revient que très peu sur les reliques, comme si le fait était de piètre importance alors même que l'évêque a autorisé en 1845 leur exposition publique certains jours de l'année ${ }^{26}$. Il donne par contre un portrait collectif de ses paroissiens :

"La très grande majorité des habitants satisfait au devoir pascal; est exacte à fréquenter les offices paroissiaux; montre de l'empressement à recevoir l'instruction religieuse; écoute la parole de Dieu avec une attention remarquée par tous ceux qui l'annoncent; aux grandes solennités il y a afflux aux confessionnaux; les communions mensuelles sont très fréquentes et très nombreuses ${ }^{27}$."

En somme la population de La Gouesnière, à en croire son recteur, est fidèle à ce que l'on attend de catholiques au milieu du XIX ${ }^{\mathrm{e}}$ siècle. C'est aussi sans doute qu'à cette date, à La Gouesnière comme dans beaucoup de paroisses du diocèse ou d'ailleurs, l'Église s'est remise de la crise révolutionnaire. La fabrique, dont la mise en route fut lente et parfois chaotique, fonctionne dorénavant de manière satisfaisante, et matériellement l'Église s'est rendue plus visible. En effet le presbytère tel que l'a connu le jeune Julien Moulin n'était guère reluisant. En 1847, à la demande d'Adolphe Baudouin, un architecte de Saint-Servan, Hippolyte Bizier La Fosse, rend compte de l'état du bâtiment sur lequel aucun travail n'a été effectué depuis quarante ans. Son diagnostic est impitoyable :

23. LAGRÉE M., Mentalités..., op. cit., p. 299.

24. Arch. dép. d'Ille-et-Vilaine, 5 V 127/1, paroisse de La Gouesnière.

25. Arch. dép. d'Ille-et-Vilaine, 6 V 145 (cité plus haut).

26. Arch. dép. d'Ille-et-Vilaine, 5 V 127/1 (cité plus haut).

27. Arch. dép. d'Ille-et-Vilaine, 6 V 146, Questionnaire de l'archevêché à MM. les curés et recteurs du diocèse de Rennes, 1861. 
" Le pignon Ouest tombe en ruine, la façade Est est lézardée en plusieurs endroits, l'élévation postérieure surplombe sensiblement, la charpente des combles est généralement très mauvaise, et la couverture qui est en paille très ancienne laisse passer l'eau de tous côtés. [...] Le presbytère est dans un état imminent de ruine qu'il y a danger réel à l'habiter ${ }^{28}$. "

Baudouin obtient de la fabrique le déblocage de fonds pour engager une rénovation complète, mais ne peut loger dans son nouveau presbytère qu'en 1852 - et encore les travaux ne sont-ils définitivement achevés qu'en 1854 -, soit l'année même où Moulin entre au séminaire à Rennes. De même le grand mouvement de restauration du bâti ou de construction d'églises neuves ne touchera le diocèse et la paroisse qu'après le départ du missionnaire pour le Canada. C'est à La Gouesnière Gilbert Vromet qui lors de son bref passage comme recteur fera entreprendre l'édification d'un nouveau clocher en 1867-1868, sans détruire pour autant l'essentiel de la structure. Et c'est son successeur qui décide d'aller de l'avant dans la dévotion mariale, dans une paroisse dont la patronne était déjà Marie mais sans que cela ne suscite jusque-là d'élan particulier. Hue, en 1835, ne signalait aucune relique de Marie, or Auguste Feuillet, avec qui Moulin correspond, fait certifier quarante ans plus tard, "deux parcelles de reliques, l'une du Voile de la Très Ste Vierge, l'autre du Manteau de St Joseph son époux ", dont l'authenticité serait prouvée par une inscription de 1775 dans le piédestal de la statue qui les renferme ${ }^{29}$.

Julien Moulin, qui maintient encore par sa correspondance un lien fort avec sa paroisse, connaît ces évolutions, mais a vécu avant que le changement ne survienne. Il a connu le temps d'une reconstruction lente des cadres de la vie religieuse, une époque durant laquelle des prêtres sans doute très peu ouverts sur le monde tentaient de former « une citadelle à défendre contre le siècle ${ }^{30}$ " dans des conditions matérielles encore précaires, et sans qu'un triomphalisme trop ostentatoire ne soit possible. C'est de ce monde assez clos, au sein duquel sa famille trouve une place notable, qu'il émerge pour, lui, envisager de plus vastes horizons. Mais ces horizons posent justement problèmes : sont-ils purement géographiques ou aussi culturels? L'éloignement volontaire dans l'espace le mènera-t-il à une remise en cause des cadres culturels qui l'ont formé?

\section{Julien Moulin, missionnaire}

Jusqu'en 1854, Julien Moulin est à l'image de Louis-François Pinagot : il n'est possible de l'atteindre qu'en procédant par cercles concentriques, en approchant au plus près ce qu'il a connu lui-même ${ }^{31}$. À partir de cette

28. Arch. dép. d'Ille-et-Vilaine, 2 O 123/11, Procès-verbal relatif à l'état de vétusté de la maison presbytérale.

29. Arch. dép. d'Ille-et-Vilaine, 5 V 127/1 (cité plus haut).

30. LAGRÉE, M., Mentalités..., op. cit.

31. CoRBIN, Alain, Le Monde retrouvé de Louis-François Pinagot : sur les traces d'un inconnu, 1798-1876, Paris, Flammarion, 1998, 336 p. 
date, c'est sa propre voix qui est accessible à l'historien, par l'intermédiaire de sa correspondance. Il est donc possible d'approcher par ce biais sa manière de dire et de construire, de mettre en jeu, ses propres identités emboîtées.

\section{Devenir prêtre}

La vocation demeure un problème historique non résolu. Il est toujours possible de procéder à des analyses statistiques du corps des prêtres, afin de déterminer leur origine sociale, leur place dans leur famille, leur niveau culturel ${ }^{32}$. En cela d'ailleurs Julien Moulin ne se distingue guère, fils d'artisan rural, d'une famille d'un niveau de richesse et de culture très honorable. Mais Julien Moulin est l'unique fils de la famille, et ses parents auraient pu attendre autre chose, espérer une reprise de l'affaire du père, ou au moins, comme ses oncles et beaucoup de ses cousins, une installation à La Gouesnière. Son choix ne fut pas celui-là, et les traces en sont réduites. Un indice peut-être, étant donnée la fréquence des transmissions du métier de prêtre d'oncle à neveu ou par des degrés de parenté plus éloignés : la paroisse a déjà donné un prêtre. Jean Delorme, apparenté à Marie Delorme, épouse de Philippe Moulin, grand-oncle de Julien fils et né en 1784 (sa mère était une Solier), est ordonné en 1812 et devient recteur d'Hirel, une commune proche de La Gouesnière, sur la Baie du Mont-Saint-Michel ${ }^{33}$. La famille a donc déjà donné un prêtre, qui a suivi une carrière des plus classiques et qui s'éteint en 1863. Peut-être faut-il voir là le modèle de Julien.

Toujours est-il que ce dernier entre au séminaire de Rennes en 1852, à 22 ans et sans qu'il soit possible de retracer son itinéraire scolaire avant cette date hormis pour noter une anomalie. Moulin, qui a fréquenté le collège ${ }^{34}$, n'a pas suivi semble-t-il le parcours désormais bien balisé du futur prêtre, puisqu'il n'a pas connu le petit séminaire de Saint-Méen-le-Grand ${ }^{35}$, et ce n'est donc pas là, lors de la visite en 1849 de l'évêque de Natchez, qui " a bien voulu adresser quelques paroles d'édification aux élèves ${ }^{36}$ ", qu'est

32. Toutes les monographies diocésaines traitent de la question. Voir pour s'orienter LAGRÉE, Michel "La monographie diocésaine et les acquis de l'historiographie religieuse française ", Études d'histoire religieuse, 61 (1995), p. 9-41. SORREL, Christian, "Échelles et espaces : le diocèse. Réflexions sur l'historiographie française contemporaine", dans PELLISTRANDI, Benoît (dir.), L'Histoire religieuse en France et en Espagne, Madrid, Casa de Velázquez, 2004, p. 225-247 et BouTRY, Philippe, " Paroisses et clergé paroissial en France ", ididem, p. 175-200.

33. Arch. dép. d'Ille-et-Vilaine, 6 V 98, registre des mutations du clergé du diocèse de Rennes.

34. Arch. dép. d'Ille-et-Vilaine 5 V 127/6, Julien Moulin à ses parents, Portage La Loge, 30 juillet 1860 .

35. Arch. dép. d’Ille-et-Vilaine, 6 V 161, Petit séminaire de Saint-Méen-le-Grand, élèves et notes 1846-1867; Arch. dép. d'Ille-et-Vilaine, 6 V 162, Petit séminaire de Saint-Méen-leGrand, examens et prix, 1824-1894.

36. Arch. dép. d'Ille-et-Vilaine, 6 V 160, Petit séminaire de Saint-Méen-le-Grand, Organisation et activités 1829-1906, journal anonyme - sans doute d'un professeur - couvrant les années 1844-1849. 
née sa vocation missionnaire. La correspondance conservée de Julien Moulin débute par deux lettres écrites du séminaire lors de sa deuxième et dernière année de présence. À la fin de celle-ci, en effet, il décide de lui-même, sans concertation apparente avec sa famille, de s'engager dans la congrégation des Oblats de Marie Immaculée, fondée en 1815 comme Société des Missionnaires de Provence par l'évêque de Marseille $\mathrm{M}^{\mathrm{gr}} \mathrm{de}$ Mazenod pour revitaliser le catholicisme régional ${ }^{37}$. Dans les années 1830 mûrit l'idée de missions étrangères et la première est fondée en 1841 dans l'est du Canada. Lorsque Moulin intègre la congrégation, elle a déjà essaimé dans l'ouest du Canada depuis 1845 mais aussi à Ceylan, en Afrique du Sud, dans l'Oregon et au Texas, et le recrutement ne pose pas de problème majeur, reflet de l'élan missionnaire plus général du catholicisme français. Moulin n'est pas le seul Rennais à emprunter cette voie oblate : cette même année 1854, Célestin Frin le suit, l'année suivante ce sont Jean-Marie Pian et Pierre-Marie Boutin qui en prennent le chemin, et en 1858 Adolphe Chatel ${ }^{38}$. Il est malheureusement impossible de savoir s'il s'agit de recrutements occasionnés par des tournées oblates pour ce faire, ainsi que procédaient à l'époque les évêques américains. Ce qui demeure sûr c'est que le diocèse de Rennes donnait depuis déjà deux décennies de nombreux missionnaires pour l'Amérique du Nord, mais en dehors du cadre congréganiste et de préférence vers l'Indiana où une filière s'était organisée ${ }^{39}$.

Moulin est donc durant l'année scolaire 1854-1855 au noviciat oblat de Notre-Dame de l'Osier, dans l'Isère, avant de rejoindre Marseille, pour achever ses études au sein de la maison-mère. Les trois lettres qui demeurent de ces années sont importantes en ce qu'elles dessinent déjà l'attitude qu'adopteront le futur missionnaire et sa famille dans leurs relations épistolaires. De Rennes ou des Alpes, Moulin invente une manière de gérer son nouveau métier dans ses diverses implications personnelles qu'il appliquera lorsqu'il sera en poste dans le Nord-Ouest canadien. D'abord dans le souci de maintenir un lien avec La Gouesnière, avec ses parents - qui insistent également pour un tel maintien - comme avec le clergé. Les lettres ellesmêmes témoignent d'un lien écrit avec son père et sa mère d'abord, et il est probable que le lot conservé en archives soit incomplet. Mais elles témoignent aussi d'échanges matériels, dans la mesure des possibilités. Bien sûr, de Rennes, les difficultés sont peu nombreuses et les échanges nombreux: de La Gouesnière partent régulièrement des pommes et des lots de draps et

37. La seule référence demeure LEFLON, Jean, Eugène de Mazenod, Evêque de Marseille, fondateur des Missionnaires Oblats de Marie Immaculée, 1782-1861, Paris, Plon, 3 vol., 1960.

38. Arch. dép. d'Ille-et-Vilaine, $6 \mathrm{~V}$ 170, registre matricule des élèves du grand séminaire de Rennes, 1848-1914.

39. LEMARIÉ, Charles, Études sur les missionnaires bretons dans le Middle West américain (contribution à l'histoire des origines du catholicisme aux États-Unis), Tome 1, M ${ }^{\text {gr }} \mathrm{J}$.-B. David (1761-1841). Les origines religieuses du Kentucky. Chez l'auteur, 1973, 251 p. Tome 2, Monseigneur Bruté de Rémur, premier évêque de Vincennes aux Etats-Unis (1834-1839). Paris, Klincksieck, 1974, 404 p. Tome 3, Les missionnaires bretons dans l'Indiana au XIXe siècle. Chez l'auteur, 1973, 484 p. 
de chemises ${ }^{40}$. De Rennes arrivent, avec Moulin, des livres pour sa filleule ou pour sa petite sœur Marie ${ }^{41}$. Des Alpes les parents espèrent aussi une trace matérielle, mais elle n'est pas arrivée, du moins pas avant novembre 1854. S'étant renseignés, ils savaient la proximité entre Notre-Dame de l'Osier et Notre-Dame de la Salette. En bon catholiques qu'ils étaient, ils avaient donc demandé des médailles à leur fils, mais ce dernier, pris par le temps très réglé du noviciat, ne peut répondre à leurs attentes ${ }^{42}$.

Mais c'est aussi avec le clergé de sa paroisse que Moulin veut garder un contact, à la fois par reconnaissance et par intégration au réseau ecclésiastique. Du grand séminaire Moulin écrit au vicaire de La Gouesnière, JeanFrançois Duval, qui est malade et mourra dans l'année, et demande à ses parents de " présenter [s] es respects " aux deux prêtres locaux. De l'Isère, de même, les premiers que Moulin cite dans ses formules finales sont le recteur et le vicaire, cette fois Jean-François Chauchix, sans qu'il soit possible de savoir dans ce dernier cas si Moulin a assisté à l'installation du vicaire avant de rejoindre le noviciat ou s'il n'en a été que mis au courant par ses parents.

Ce lien maintenu toujours avec le point de départ sert à atténuer, pour tous les protagonistes, les souffrances de la séparation. Celles-ci ne peuvent s'exprimer toujours dans une correspondance emplie de formules qui se répètent souvent d'une lettre à l'autre et qui empruntent l'essentiel de leur contenu à la morale catholique du sacrifice et des vertus de la douleur, d'autant plus que Moulin est engagé dans ses études et entre dans son rôle de prêtre. De Rennes, il pouvait encore dire sans arrière-plan religieux que six mois de séparation " [lui] paraissai[en]t bien long[ $s]^{43}$ " quand il pensait à sa famille. De Notre-Dame de l'Osier, le novice tient un autre langage, qui est déjà celui du missionnaire canadien :

" J'ai été heureux de recevoir votre lettre et d'apprendre votre résignation. De douces larmes ont sillonné mes joues à la lecture de cette lettre. J'ai remercié la très sainte et immaculée Vierge Marie de m'avoir obtenu cette précieuse faveur, la priant de nouveau d'adoucir vos sacrifices. Pèlerins sur la terre, nous ne devons pas nous attacher aux choses d'ici-bas mais nous devons porter nos regards vers le ciel qui est notre véritable patrie près à tout sacrifier pour notre Dieu qui saura bien nous dédommager des quelques sacrifices que nous faisons pour son amour. Quoique je sois séparé de vous n'allez pas croire que je vous oublie. Je prie pour vous. Je prie la consolatrice des affligés de vous consoler ${ }^{44}$."

Comment ne pas lire là à la fois l'intégration par Moulin de l'enseignement donné au séminaire comme au noviciat, l'intégration de son nouvel

40. Arch. dép. d'Ille-et-Vilaine, 5 V 127/6, Julien Moulin à ses parents, Rennes, le 6 février 1854.

41. Arch. dép. d'Ille-et-Vilaine, 5 V 127/6, Julien Moulin à ses parents, Rennes, le 9 avril 1854.

42. Arch. dép. d'Ille-et-Vilaine, 5 V 127/6, Julien Moulin à ses parents, Notre-Dame de l'Osier, le 20 novembre 1854.

43.9 avril 1854 (cité plus haut).

44. 20 novembre 1854 (cité plus haut). 
état, mais aussi une réelle souffrance, de son côté comme du côté de sa famille. Chacun vit une séparation, le fils unique est parti, perdu pour La Gouesnière, mais il faut trouver la consolation dans la foi, dans la conviction de l'utilité de son destin.

Ce sentiment douloureux peut être renforcé par le choc de l'étranger, vécu là encore par Moulin comme par ses parents, sur le mode tant de la curiosité que de l'inquiétude. Les parents sont dans une situation d'ignorance et désirent savoir l'environnement de leur fils. Les Alpes leur semblent de ce point de vue totalement étrangères et Moulin, comme il le fera plus tard pour le Canada, leur décrit brièvement les lieux : « Notre-Dame de Lozier est située sur une montagne qui est entourée elle-même de montagnes qui sont des deux côtés et où on commence à voir la neige qui couvre ces montagnes pendant une partie de l'année ${ }^{45}$. " Tout est dit de l'exotisme de l'Isère. Ce sentiment d'éloignement du fait de la méconnaissance des lieux suscite des interrogations. La santé du séminariste est l'objet de toutes les attentions parentales, même à Rennes puisque le climat, moins littoral et plus urbain, est donc moins sain semble-t-il dans l'imaginaire des parents Moulin. Le fils peut les rassurer : "Depuis la rentrée je n’ai pas encore eu d'indisposition, ce qui fait voir que le climat de Rennes m'est bien sain ${ }^{46}$. " Des Alpes il répond de même que sa santé ne pose pas de problème mais les représentations de la montagne et de l'étranger travaillent la famille Moulin.

Il faut alors en passer par le familier pour apprivoiser le lointain. Mais Julien Moulin ne commence à adopter cette tactique discursive, dont on suppose qu'elle lui sert autant à lui qu'elle doit être utile à ses yeux pour rassurer la famille, qu'une fois hors de France. Ainsi en 1857, jeune Oblat, est-il à Dublin. Il doit décrire une chapelle : " Le saint sacrement était dans une espèce de niche un peu assez semblable à celle de Château-Malo où se trouve la sainte Vierge ${ }^{47}$. "Six mois plus tard il débarque à Québec et se rend directement chez les Oblats locaux pour y dire une messe : "Je me retrouvais là comme dans notre vieille Bretagne, je fus bien édifié de la piété des fidèles de cette contrée ${ }^{48}$. " Il s'agit pour Moulin de comparer objets et hommes, de les rapprocher de la réalité de La Gouesnière afin de faire comprendre l'étrangeté du monde en la ramenant à une distance culturelle si possible faible, et donc d'atténuer les angoisses d'un éloignement qui n'est pas uniquement géographique.

\section{Le métier de missionnaire}

Les études concordent, même si les travaux sur le sujet demeurent insuffisants, pour porter un jugement sévère sur la formation offerte dans

45. Ibidem.

46. 6 février 1854 (cité plus haut).

47. Julien Moulin à ses parents, Dublin, 25 décembre 1857.

48. Julien Moulin à ses parents, Montréal, 24 juin 1858. 
les grands séminaires à l'époque de Julien Moulin. Les histoires diocésaines traitent toutes des établissements d'où sortent les cadres paroissiaux. Même lorsque l'évêque est de la stature intellectuelle d'un M Mrr Dupanloup à Orléans, le séminaire est d'une grande pauvreté, ne dispense que des " connaissances [...] ingurgitées sans souci de culture, [...] [sans] aborder les problèmes qui se posent à la pensée contemporaine. Cette éducation en vase clos ignore le monde moderne ${ }^{49}$ ". Il semble que ce soit là un portrait digne d'être généralisé, et que la formation sacerdotale privilégie alors l'obéissance, l'humilité, le silence, la prière et la méditation religieuse, loin du monde que les séminaristes auront à affronter et dominer à leur sortie $^{50}$. Le grand séminaire de Rennes ne fait pas exception malgré les quelques améliorations apportées après une sorte d'aggiornamento en $1843^{51}$. Les bâtiments en tout cas ne sont guère reluisants, et ce n'est qu'après le départ de Moulin qu'un nouvel établissement verra le jour. C'est là qu'on lui enseigne un modèle, une " ayance ", pour reprendre le terme utilisé par Paul Airiau, c'est-à-dire que « l'enjeu de la formation ecclésiastique est bien de donner une identité ad extra, qui n'est possible que si toute la personnalité est saisie ${ }^{52}$ ". Les séminaristes participent de cette construction d'un nouveau soi par des pratiques inédites pour eux, notamment l'introspection par l'oraison méthodique, et sortent de leurs études très conscients d'une identité collective forte, qui coupe les jeunes prêtres non seulement de leur milieu d'origine, qui en fait donc des déracinés culturels, mais aussi de l'ensemble du monde profane, ce qui potentiellement les isole dans leurs paroisses d'affectation.

En conséquence, "l'identité sacerdotale est foncièrement instable " car dans la confrontation avec le monde le prêtre se heurte à ses habitudes acquises, qui ne le préparent nullement à son métier, qui est fait de relation et non de fermeture. L'idéal - la construction d'un modèle du prêtre irréprochable - se heurte au réel de la paroisse. La solution qui consiste à reproduire dans sa cure le rythme de vie du séminaire, organisé autour de la prière, des exercices de piété ${ }^{53}$, ne peut être tenable à long terme. Une autre solution réside dans la création des congrégations, comme celle des Oblats de Julien Moulin. Une congrégation spécialisée, ici dans la mission étrangère, développe d'autres habitudes, d'autres normes a priori destinées à approfondir cette question du rapport du prêtre au monde. En ce sens, ces congrégations, nées d'un désir de reconquête catholique contre la

49. Marcilhacy, Christiane, Le Diocèse d'Orléans sous l'épiscopat de $M^{g r}$ Dupanloup, 18491878, Paris, Plon, 1962, p. 95.

50. LaunAY, Marcel, Les Séminaires français aux XIX et $X X^{e}$ siècles, Paris, Le Cerf, 2003, $261 \mathrm{p}$.

51. LAGrÉE, M., Mentalités..., op. cit.

52. AIRIAU, Paul, " La formation sacerdotale en France au XIX ${ }^{\mathrm{e}}$ siècle ", Archives des sciences sociales des religions, 133 (janvier-mars 2006), p. 37. Les lignes qui suivent s'inspirent largement de ses analyses. Voir du même "Les séminaires diocésains français 1880-1914 ", Revue d'histoire de l'Église de France, 91 (janvier-juin 2005), p. 71-89.

53. LaUnAY, Marcel, Le bon prêtre. Le clergé rural au XIXe siècle. Paris, Aubier, 1986, 326 p. LEMAîTRE, Nicole (dir.), Histoire des curés, Paris, Fayard, 1994, 532 p. 
modernité, introduiraient aussi cette dernière dans l'Église par une certaine adaptation critique aux temps nouveaux ${ }^{54}$.

Julien Moulin, lorsqu'il doit officier dans son poste missionnaire de L'Île-à-la-Crosse et dans les postes alentours, situés dans les actuels Saskatchewan et Manitoba, est le fruit de cette double formation, celle des séminaires et celle des congrégations. Ce qu'il dit de son métier, de son quotidien, dans les lettres qu'il adresse à ses parents mais aussi en une occurrence à son parrain et à un " président " dont l'identité est inconnue, révèle en fait la culture du missionnaire, c'est-à-dire la confrontation de l'ayance et du monde, mais à travers le filtre de ce qu'il veut bien en dire. Il ne faut en effet pas confondre la parole de Moulin avec une quelconque " vérité " au prétexte qu'il s'agirait d'un témoignage de première main relevant de l'intime. Moulin, comme tout migrant, met en œuvre des stratégies discursives et ne rapporte à ses correspondants que ce qu'il juge important, en fonction des attentes qu'il croit percevoir et de l'image qu'il se fait de son sacerdoce et de ses difficultés ${ }^{55}$.

Ainsi son métier tel qu'il le décrit au premier abord s'éloigne fort de ce qui a pu lui être enseigné en France. Il semble être caractérisé par quatre traits majeurs : l'isolement, la barrière linguistique, le travail de conversion et la mobilité. L'isolement est réel. De Montréal Moulin est passé par les États-Unis pour, depuis Saint-Paul dans le Minnesota, suivre une caravane de Métis jusque Saint-Boniface dans ce qui était alors le Territoire de la Baie d'Hudson, administré par la compagnie éponyme. Un an après son arrivée, Moulin est envoyé par $M^{\text {gr }}$ Taché ${ }^{56}$ sur son poste de L'Île-à-la-Crosse, à 40 ou 45 jours de là, en canot, en traîneau ou à pieds. L'éloignement est d'autant plus mal ressenti que les liaisons postales sont rares : "Il n'y a que deux occasions chaque année d'expédier les lettres pour le Nord à partir de la Rivière Rouge ${ }^{57}$. " Cet éloignement de la famille et de l'autorité - le siège épiscopal de Saint-Boniface avant qu'il en soit créé un à SaintAlbert - ne signifie pas absence vie relationnelle mais il est vécu comme un isolement.

Les populations dont Moulin a la charge ne contribuent pas à le briser. La communication avec les " païens " est un des problèmes auxquels ont été confrontés tous les missionnaires en terre étrangère, et auquel une réponse a été apportée tôt, dès le XVI ${ }^{\mathrm{e}}$ siècle : l'apprentissage des langues est une

54. Voir aussi Cabanel Patrick, "Les congrégations religieuses ", dans Pellistrand, Benoît, L'histoire..., op. cit., p. 249-270.

55. GERBER, D. A., "Acts of deceiving and withholding in immigrant letters : personal identity and self-presentation in personal correspondence ", Journal of Social History, 39, 2, p. 315-330 et plus généralement Authors of their lives : the personal correspondence of British immigrants to North America in the nineteenth century, New York, New York University press, 2006, $424 \mathrm{p}$.

56. Sur l'évêque et son rôle dans la construction de cette Église, HuEL RaYmond, J. A., Archbishop A.-A. Taché of St. Boniface : The "Good fight" and the Elusive Vision, Edmonton, University of Alberta Press, 2003, 429 p.

57. Julien Moulin à ses parents, Île-à-la-Crosse, 20 juillet 1863. 
donnée essentielle de la mission. Mais la tâche est ardue. Moulin est long à pouvoir travailler véritablement au contact des Indiens Chipewyans ${ }^{58}$ et Cris dont il a la charge. Le 28 mai 1862, trois ans et demi après son arrivée, il affirme encore: "J'ai commencé à entendre les confessions dans cette dernière langue [le chipewyan], mais avec beaucoup de difficultés ${ }^{59}$. " En 1867, les choses ont changé : "Il m'est arrivé d'entendre en confession parfois 3 personnes qui parlent 3 langues différentes ${ }^{60}$. " En 1869, depuis longtemps chef du poste, il trouve pénible d'avoir avec lui un jeune prêtre " qui ne connaît pas les langues ${ }^{61}$ ". Aurait-il oublié le temps qu'il lui a fallu lui-même pour résoudre le problème?

Sans parler la langue, le travail missionnaire est inefficace, car la mission est un art de la parole. Il faut expliquer le dogme, tant lors des prêches que lors des instructions données le matin comme l'après-midi, aux enfants comme aux adultes, et le mettre en pratique : « j'ai baptisé 13 enfants fait un mariage, entendu 109 confessions, administré 25 communions ${ }^{62}$ " explique Moulin dans la logique comptable qui caractérise la mission puisque celleci est une conquête des âmes, qui doit donc être mesurable précisément et dont les résultats doivent être exposés. Cette œuvre d'inclusion de populations " encore assises dans les ténèbres de la mort ${ }^{63}$ " au sein de l'Église fait la spécificité de Moulin - qui gère aussi des populations déjà catholiques, mais dont ce ne pas le métier principal - par rapport au prêtre du pays malouin qu'il aurait pu devenir. Elle nécessite une mobilité constante, pour deux raisons : la superficie du territoire à couvrir d'une part, le nomadisme des populations cibles d'autre part. Aussi le site de l'̂lle-à-la-Crosse n'est-il à l'arrivée de Moulin, et pour quelques années encore, qu'un camp de base. Situé à proximité d'un poste de la Compagnie de la Baie d'Hudson à dessein, il permet d'accueillir deux fois par an les Indiens qui viennent vendre leurs fourrures, et de rayonner pour faire des missions de quelques semaines ou quelques mois vers des villages éloignés. Moulin quitte ainsi son poste le 26 septembre 1865 pour une mission au Portage La Loche, à 6 jours de canots de là. Près de deux mois plus tard il est de retour à l'Île-à-la-Crosse pour en repartir immédiatement pour Fort Carillon où il arrive le 7 décembre après deux semaines de voyage. De retour à sa mission, il en repart le 22 mai 1865 pour le Lac Caribou, sa nouvelle affectation. L'été de cette année est donc consacré à un circuit afin de passer à Norway House pour y rencontrer les autorités de la Compagnie afin de discuter ravitaillement et à Saint-Boniface pour y rencontrer $\mathrm{M}^{\text {gr }}$ Taché. Enfin, le 26 février 1867, Moulin était rappelé à l'Île-à-la-Crosse, en tant que responsable du poste.

58. Que les missionnaires appellent à l'époque Montagnais, qu'il ne faut donc pas confondre avec les Montagnais de l'est canadien.

59. Julien Moulin à ses parents, Île-à-la-Crosse, 28 mai 1862

60. Julien Moulin au "président ", Île-à-la-Crosse, 6 mai 1867.

61. Julien Moulin à ses parents, Île-à-la-Crosse, 5 août 1869.

62. 28 mai 1862, (cité plus haut).

63. Julien Moulin à ses parents, Montréal, le 30 juin 1858. Il s'agit des paroles du supérieur des Oblats de Montréal lors de la cérémonie de départ de Moulin pour la mission. 
Il n'aura cessé de se déplacer depuis un an et demi, dans des conditions souvent pénibles, de lenteur et de froid.

En conséquence, Julien Moulin aurait vécu une vie exceptionnelle, qu'aucun prêtre d'aucune paroisse rurale française ne pouvait envisager de vivre. Le séminaire ne l'y préparait pas, il avait fallu s'y adapter, apprendre les langues et les manières de faire du pays, apprendre à rencontrer des peuples inconnus. Mais il ne faut pas surévaluer les différences entre le modèle sacerdotal appris en France et le métier de missionnaire. Ainsi l'instabilité extrême, l'état de tension inhérent à la condition sacerdotale et poussée à l'extrême chez les missionnaires du fait de leur contact particulier au monde peut se résoudre aussi par une quête de conformité.

Certes il est difficile de nier que l'éloignement peut être évité si le prêtre choisit une carrière plus classique. L'idéal étant même le rapprochement, le missionnaire se place hors du modèle. Mais son isolement n'est pas hors norme. D'abord parce que son statut et ses habitudes le coupent de ses paroissiens et que tel est le principe qui préside à son identité sacerdotale : il est à part. Même nommé dans un milieu rural proche de celui de ses origines, Moulin aurait pu, comme nombre de prêtres, vivre isolé. La mission a poussé cette logique à l'extrême, mais en reconstruisant dans le même temps des réseaux de sociabilité, entre membres de la congrégation, ou entre " civilisés " face aux " sauvages ". La question linguistique se pose avec acuité en certaines paroisses bretonnes - le diocèse voisin de celui de Julien Moulin, celui de Saint-Brieuc, était bilingue, français et breton - et l'Église a souvent mené une politique de la langue. S'attacher à la langue vernaculaire des fidèles est de ce point de vue un combat de prêtre breton aussi bien que de missionnaire au loin ${ }^{64}$.

De même, les tâches du missionnaire, si elles sont spécifiques dans leur mise en œuvre, ne le sont pas dans leur répétition et dans leur caractère obligatoire. Instruire, dire la messe, confesser, célébrer baptêmes et mariages, le tout selon des rites établis depuis Rome : à La Gouesnière ou à l'Île-à-la-Crosse, le métier est identique dans ses étapes obligées. C'est que l'Église a vocation universelle et a comme objectif de reproduire un modèle, variable dans le temps certes mais qui ne doit a priori pas l'être dans l'espace. Les consignes de $\mathrm{M}^{\mathrm{gr}}$ de Mazenod aux Oblats étaient en ce sens d'une grande clarté : il s'agissait de transformer, lentement, le quotidien et la culture des Indiens en les poussant vers la sédentarisation afin de créer des communautés catholiques stables. L'île-à-la-Crosse avait alors vocation à devenir, d'une certaine manière, dans les mains de Julien Moulin, une paroisse du pays malouin.

L'école a, dans ce cadre, un rôle fondamental à jouer. À partir de son retour à l'Île-à-la-Crosse en 1867, Moulin n'a de cesse d'en entretenir ses correspondants. Les Sœurs Grises venues de Montréal ont en charge l'ac-

64. LAGRÉE, M. Religion et culture en Bretagne, 1850-1950, Paris, Fayard. Lagrée M. (dir.), Les parlers de la foi : religion et langues régionales, Rennes, PUR, 1995, $161 \mathrm{p}$. 
culturation des jeunes Indiens, leur transformation en "civilisés ", donc avant tout en bons chrétiens, et dans le même temps en paysans, tant est idéalisée une sorte de civilisation rurale immobile et merveilleusement catholique. Il y a bien volonté de reproduction. En 1869, Moulin accueille dans sa mission $M^{\text {gr }}$ Grandin. C'est lui qui célèbre la Fête-Dieu :

\begin{abstract}
" Nous avons eu la procession de la fête Dieu à laquelle ont assisté tous les sauvages ils ont gardé un ordre parfait pendant tout le temps de la procession. Ils étaient tous sur deux rangs. Pendant le temps de la procession on a chanté des cantiques dans les deux langues. Le st sacrement était porté par $\mathrm{M}^{\mathrm{gr}}$ Grandin. Le dais était porté par des engagés de la compagnie et des sauvages. Il y a avait un certain nombre de petites filles de l'école qui étaient habillées en blanc. Les petits garçons portaient des oriflammes ou petits drapeaux. Il y avait deux fleuristes et un thuriféraire; les autres portaient les insignes épiscopaux. On a fait trois reposoirs. On avait coupé des arbres qu'on avait plantés des deux côtés du chemin. L'un des reposoirs se trouvait en haut d'une montagne ou est planté la croix de la Mission. Cette procession a laissé un précieux souvenir que nos bons sauvages n'oublieront pas ${ }^{65}$."
\end{abstract}

Entre le respect des rites, la magnificence tentée malgré les difficultés, l'affichage d'unanimité de la communauté et du succès de l'instruction des enfants, le respect et l'admiration dus à l'évêque, la présence de la croix de Mission si importante au XIX ${ }^{\mathrm{e}}$ siècle, Moulin donne là un tableau d'une chrétienté idéale qui ne s'éloigne guère, finalement, de celui que donnaient dans leurs réponses aux questionnaires épiscopaux les recteurs de la Gouesnière. Il y a chaque fois un souci profond de normalité du monde. C'est dans ce travail de normalisation idéale, toujours rêvée mais jamais achevée, que Moulin trouve matière à vivre son isolement, son éloignement, la répétition de ses gestes professionnels, la tension inhérente à son état.

\title{
Le fils de La Gouesnière
}

Julien Moulin, dans sa situation missionnaire, doit gérer à la fois l'instabilité de sa condition de prêtre et sa nature de simple migrant. Car malgré la spécificité de sa profession et des motifs de son départ, son acte est bien avant tout migratoire. S'il ne s'inscrit pas dans de classiques réseaux migratoires familiaux ou locaux, comme beaucoup de membres du clergé diocésains de l'Ouest français (vers l'Indiana notamment) ou de l'Ain (vers le Minnesota), c'est que sa congrégation, les Oblats de Marie Immaculée, constitue en soi son propre réseau. C'est au sein de celle-ci que l'insertion sociale se fait dans le pays d'arrivée.

Moulin est donc confronté à des difficultés que tout migrant peut rencontrer et y apporte des réponses que d'autres ont également apportés dans des cas similaires, notamment dans la relation à ceux qui sont restés au pays. L'Ouest canadien fait craindre le pire, a priori. Il semble qu'il faille ici distinguer entre les craintes dues à l'horizon d'attente des Moulin et

65. Julien Moulin à ses parents, Île-à-la-Crosse, 13 juin 1869. 
celles dues à ce que Julien rapporte de son nouveau pays. Car ce sont d'abord aux Indiens que les missions nord-américaines sont liées dans l'imaginaire français, avant toute considération environnementale. Les premières nouvelles sont alarmantes : le jeune Oblat écrit qu'il va au loin prendre en charge des tribus païennes et sa route est interminable, elle semble le mener au bout du monde, vers les terres sauvages de l'intérieur du continent américain. Il faut même emprunter une caravane de Métis pour s'y rendre et se livrer à des rites qui ne peuvent qu'angoisser ceux qui restent au pays : "Il n'y avait plus rien à craindre des sauvages car nous avions une brigade composée de plus de 130 charrettes. [...] Le soir on commença à former le rond avec les charrettes et à renfermer les chevaux dedans ${ }^{66}$. " Circuler en nombre, faire le cercle, le tout afin d'éviter une agression indienne : les récits qui circulent depuis longtemps déjà en France sur ces Indiens génériques trouvent ici un écho angoissant. Moulin doit rassurer dès la lettre suivante :

"D'après la lettre que vous m'avez envoyée vous croyez que je suis à tout moment sur le point de tomber entre les mains des sauvages pour en recevoir les plus mauvais traitements mais rassurez-vous encore cette fois car il n'en est point ainsi. Je puis vous dire que depuis quarante ans qu'on a commencé ces missions dans le Nord de l'Amérique aucun n'a été tué par les sauvages, je dirais même maltraité ${ }^{67}$."

L'année suivante Moulin prend lui-même en charge une mission indienne et ce qu'il ne cessera d'en dire rassurera pour de bon sans doute car il ne sera plus jamais question du problème. Il en va de même avec le froid, mais sur une période légèrement plus longue. Le 22 janvier 1859, en même temps qu'il précise avoir à faire à des Indiens inoffensifs, il note :

"Quant au froid quelque rigoureux qu'il soit, on a point à en souffrir et je dirais même qu'on en souffre beaucoup moins qu'en France car le bois est abondant dans ce pays. La neige à la vérité reste sur la terre à peu près cinq mois de l'année, mais c'est le meilleur temps pour voyager; car la neige est durcie par le froid, ensuite on a des voitures sans roues qui glissent fort bien sur la neige. [...] Tout ce que je puis dire c'est que je suis arrivé au milieu de l'hiver sans avoir d'engelure aux mains ce que j'avais toujours éprouvé en France ${ }^{68}$."

Puis Moulin fait connaissance avec les raquettes, qui présentent dans la relation avec La Gouesnière un double avantage. Elles permettent de dire le confort qu'il est possible d'avoir en ces contrées lointaines (" je ne fatiguais pas avec ces raquettes tandis que sans ces raquettes je ne pouvais pas voyager ${ }^{69}$ ") et elles offrent la chance de pouvoir fournir des développements sur les coutumes locales, sur l'exotisme. Ces simples objets du quotidien se font, dans la lettre que Moulin adresse à son parrain en mai 1866,

66. Julien Moulin à ses parents, été 1858.

67. Julien Moulin à ses parents, Saint-Boniface, le 22 janvier 1859.

68. Ibidem.

69. Julien Moulin à ses parents, Île-à-la-Crosse, le 15 janvier 1860 . 
le symbole du Canada et ses hivers, objets matérialisés par un dessin, le seul présent dans la correspondance du missionnaire ${ }^{70}$.

Donc le froid, s'il est souvent qualifié d' " excessif ", n'est pas un obstacle : "Je suis en parfaite santé et mes voyages loin de nuire à ma santé la rendent meilleure ${ }^{71}$. " La correspondance constitue ici un filtre particulièrement efficace à l'expression des sentiments de Moulin. À l'en croire, l'Îleà-la-Crosse, poste situé à la limite entre la Prairie et la forêt boréale, relève presque du pays de cocagne. Les missionnaires, ravitaillés de manière insuffisante par la Compagnie de la Baie d'Hudson et leurs autorités religieuses, créent des champs et élèvent un troupeau. Moulin donne à plusieurs reprises des chiffres précis de ses récoltes, mais dans un objectif unique : "J'ai voulu vous donner ces quelques détails afin de vous empêcher de vous apitoyer sur mon sort ${ }^{72}$. "C'est que, alors que la région de la Rivière Rouge est ravagée par les sauterelles, "nous sommes traités en enfants gâtés par la divine Providence qui se charge de nous pourvoir abondamment dans nos froides contrées du Nord ${ }^{73}$ ".

De surcroît, tout dans le Nord-Ouest canadien peut être ramené à une réalité bretonne ou même malouine. Une fois de plus, une pratique discursive fréquente de Moulin peut avoir deux interprétations. L'utilisation de références issues de son pays d'origine peut servir à faire comprendre, par la comparaison, le Canada, mais elle révèle aussi la permanence de l'identité du missionnaire. D'autant plus que cet usage s'épuise au fil du temps comme si Moulin avait fini par ne plus pouvoir penser son monde en des termes bretons mais uniquement en fonction des réalités locales. Ce qui n'empêche pas le maintien du lien affectif avec le pays et ses habitants, mais qui marque une sorte d'intégration par absorption des références culturelles locales, à partir de la fin des années 1860, soit au bout de dix ans de présence missionnaire.

En 1859, Moulin n'en est pas là. Les pommes de terre de la Rivière Rouge sont alors à comparer avec les pommes de terre bretonnes ${ }^{74}$, et les bateaux utilisés sur les rivières et les lacs canadiens sont similaires à ceux " qui traversent [la Rance] à Dinan ${ }^{75}$ ". Mais Moulin sait aussi faire sentir la connexion des deux mondes, le nouveau, le sein, et l'ancien, celui de ses parents :

" Il me semble que vous demandez ce que c'était que la Compagnie de la Baie d'Hudson. [...]. Cette compagnie se compose de marchands de Londres qui envoient des agents parmi les sauvages pour traiter avec eux les fourrures, c'est-à-dire les peaux des animaux qu'ils tuent à la chasse. Ces peaux se vendent excessivement cher en France et en Angleterre. Ainsi vous voyez

70. Julien Moulin à son parrain, Lac Caribou, 29 mai 1866.

71. Julien Moulin à ses parents, Île-à-la-Crosse, le 28 mai 1862.

72. Julien Moulin à ses parents, Île-à-la-Crosse, le 7 janvier 1863.

73. Julien Moulin à ses parents, Île-à-la-Crosse, le 13 juin 1869 .

74. 22 janvier 1859, ididem.

75. Julien Moulin à ses parents, Saint-Boniface, le 6 juin 1859. 
très souvent à St Malo et à St Servant des dames porter des colliers de poils de même que leurs manchons. La plupart de toutes ces fourrures sont expédiées par la Compagnie de la Baie d'Hudson. [...] Ils montent chaque année une vingtaine de bateaux aussi grands que les bateaux de Dinard ou ils mettent jusqu'à 80 paquets de 90 livres chacun ${ }^{76}$. "

En sus du procédé comparatif habituel, Moulin construit un lien entre le Nord-Ouest canadien et le pays malouin. De même qu'il tente ainsi de projeter le Canada en Bretagne, il veut retrouver l'Ouest français au Canada en y cherchant et en y notant les missionnaires qui auraient des origines proches des siennes. Il n'en manque pas, l'Ouest français étant grand pourvoyeur de vocations. Ainsi au Lac Caribou, en 1865, Moulin se trouve en compagnie d'un prêtre du diocèse de Laval et d'un frère de celui de SaintBrieuc $^{77}$. Le petit pays peut donc être reconstitué. Et même si Moulin est sans doute, comme beaucoup de migrants, en quête de ces réseaux culturels qui permettent de se construire une vie acceptable en terre étrangère, il s'agit aussi, là encore, de montrer aux parents qu'il n'y a à craindre ni solitude ni isolement.

Au demeurant Moulin ne tient pas plus à être isolé au cœur du Canada que vis-à-vis de La Gouesnière. Sa correspondance en atteste, de même que le souci qu'il continue d'avoir de sa famille élargie, dont ses parents continuent de l'entretenir. La nouvelle du décès de son oncle Michel Moulin et de sa femme ${ }^{78}$ ou plus tard le destin de certains de ses cousins ${ }^{79}$, s'il faut l'en croire, le touchent encore alors que cela fait plus de dix ans dans un cas, vingt dans l'autre, qu'il n'a rencontré de membre de sa famille. Encore que des rencontres, il y en eu, mais de manière indirecte.

En effet, si Julien Moulin lui-même ne rentre qu'une fois à La Gouesnière, en $1899^{80}$, de prestigieux émissaires se chargent de la transmission des nouvelles et des objets. Les évêques du Nord-Ouest canadien sont à cette époque tous des Oblats, et ils doivent se rendre régulièrement en Europe pour se faire consacrer d'abord, pour des raisons administratives diverses ou afin de se rendre en 1870 à Rome pour le concile. Or ces évêques profitaient de leur séjour français pour entretenir les réseaux de leurs confrères Oblats. Ainsi la première lettre dans laquelle Moulin annonce à ses parents qu'il ne reste pas à Saint-Boniface mais s'en va rejoindre son poste à l'Île-à-la-Crosse est envoyée de France par $\mathrm{M}^{\mathrm{gr}}$ Grandin qui vient d'être nommé coadjuteur de $\mathrm{M}^{\mathrm{gr}}$ Taché et qui est rentré de ce fait brièvement en Europe. Il annonce de même aux parents Moulin qu'il est prêt sur son trajet retour à convoyer " les lettres et autres objets que vous voudrez remettre au père Moulin ${ }^{81}$ ". En 1865, c'est $M^{\text {gr }}$ Faraud qui va à La Gouesnière rencontrer la famille : « J'ai

76. 20 juillet 1863 (cité plus haut).

77. Julien Moulin à ses parents, Lac Caribou, 29 mai 1866.

78. Julien Moulin à ses parents, Île-à-la-Crosse, 7 janvier 1869.

79. Julien Moulin à son père, Lac Muskey, 29 décembre 1878.

80. Arch. dép. d'Ille-et-Vilaine, 5 V 127/1 (cité plus haut).

81. 6 juin 1859, op. cit., annotation de Grandin en marge. 
eu l'avantage de revoir $M^{\text {gr }}$ Faraud qui m'a parlé de longuement de vous a qui a été très édifié de votre résignation à la sainte volonté de Dieu ${ }^{82}$." En 1868 encore, $M^{\text {gr }}$ Grandin est en France : il passe par La Gouesnière et y prend des lettres et des objets, s'entretient avec les parents : " Il m'a parlé de vous et de votre bonheur j'ai tous les détails de votre entrevue avec lui et j'ai été heureux moi-même ${ }^{83}$. "Le passage de $M^{\text {gr }}$ Clut est espéré par Moulin l'année suivante ${ }^{84}$, lors de son voyage au concile, mais aucune certitude ne peut être apportée à ce sujet. Par contre Grandin, qui semble avoir développé des liens privilégiés avec Julien Moulin, retourne à La Gouesnière en 1878. Ses passages ne sont pas documentés, mais il est probable que la visite d'un évêque d'un diocèse bien marginal mais aussi très exotique ait été un événement dans la commune.

En 1868, Grandin apporte avec lui non seulement les lettres de la famille mais aussi des portraits que le missionnaire reçoit " avec bonheur ${ }^{85}$ ". Il y voit aussi une obligation de réciprocité mais ardue à remplir. La solution s'impose, Moulin envoie à ses parents des présents que d'autres lui ont fait :

" Je vous envoie deux chapelets dont l'un a été béni par le pape, il est fait avec des noyaux d'olive, il m'a été donné l'année dernière par Monseigneur Grandin. L'autre est un chapelet en pierre qui m'avait été donné, il y a dix ans, par un Missionnaire qui se trouve dans le poste le plus reculé au Nord je m'en suis servi depuis ce temps. J'aime à croire lorsque vous prierez dessus vous ne m'oublierez dans vos prières ${ }^{86}$."

Il y a là le double signe d'une large circulation de l'objet de dévotion à l'intérieur même de l'institution, jusqu'à des mouvements transatlantiques qui font revenir le chapelet romain acquis en 1868 par $\mathrm{M}^{\mathrm{gr}}$ Grandin en Europe auprès d'une famille de La Gouesnière. Les Moulin, dans leur paroisse, possèdent un chapelet quasi pontifical ainsi qu'un témoignage du travail de conversion sur les marges les plus extrêmes du monde. L'acte de Moulin est essentiel en ce qu'il offre une fenêtre sur un univers culturel, fait d'une triple obédience en quelque sorte, à Rome, à son petit pays, et à ses terres d'adoption et de sacerdoce. Il vit avec cette triple identité. Mais dans ses pérégrinations quotidiennes, Rome et La Gouesnière sont loin. Il a apprivoisé le Nord-Ouest canadien et ses populations.

\section{L'univers relationnel du missionnaire : la construction des identités}

Il faut à tout migrant, et donc à tout missionnaire, reconstruire sur les nouveaux lieux de vie et de travail des repères identitaires permettant de se situer dans le monde. Il faut donc pour construire sa propre iden-

82. Julien Moulin à ses parents, Norway House, 12 juillet 1865.

83. Julien Moulin à ses parents, Île-à-la-Crosse, 7 janvier 1869.

84. Julien Moulin à ses parents, Île-à-la-Crosse, 13 juin 1869.

85. 7 janvier 1869 (cité plus haut).

86. 13 juin 1869 (cité plus haut). 
tité neuve construire celle des autres, rendre cohérent l'univers a priori inconnu auquel on se trouve confronté. Il s'agit avant tout d'une opération de classement des populations auxquelles le migrant à a faire, afin de reconstituer sous ses yeux un fonctionnement social compréhensible dans lequel jouer son propre rôle. Le monde social du Nord-Ouest canadien tel que le dit Julien Moulin se divise en trois grandes catégories, trois cercles concentriques : le clergé et les sœurs d'abord; les autres " civilisés " ensuite (employés de la Compagnie de la Baie d'Hudson et missionnaires concurrents); les ouailles enfin, avec une distinction fine entre Indiens et Métis.

Le 8 janvier 1864, Moulin avoue à ses parents : "Vous avez appris que je suis resté seul cette année. Il y a plus de six mois que je n'ai pas vu de prêtre. J'ai du faire seul la Mission d'Automne ». Mais peu après il ajoute :

" Je vais deux fois par semaine au fort qui se trouve à une demi-lieue d'ici pour y faire le catéchisme [...]. Je suis obligé d'avoir l'œil à tout. J'ai 4 hommes engagés que je suis obligé de nourrir et de chauffer et même d'entretenir leur famille. J'ai aussi deux frères convers avec moi. Ce sont les sœurs au nombre de deux avec une sœur converse, qui nous lavent et qui nous préparent nos aliments. Je dois en outre avoir soin des enfants de l'école qui sont au nombre de treize ${ }^{87}$."

Il y a là un paradoxe qui n'est qu'apparent : la solitude de Moulin au milieu de tant de personnes aux identités multiples. C'est bien en fait là que le bât blesse. Moulin souffre de la solitude car il est seul de son état. L'absence de prêtre, d'égal culturel et statutaire, lui pèse et révèle à quel point le reste de l'humanité de l'Île-à-la-Crosse lui demeure extérieur. À commencer par les autres membres de l'Église. Les frères converts qui se succèdent au poste restent dans la correspondance de Moulin éternellement dans l'ombre, au contraire des prêtres. La hiérarchie interne à l'institution se lit dans le souci de nommer dans un cas, de l'oubli du nom et donc du peu de cas fait de l'individu, dans l'autre. Il en résulte peut-être des tensions sur le terrain, d'autant que tous vivent parfois sous le même toit dans une cohabitation obligatoire mais non choisie ${ }^{88}$. Les sœurs, elles, n'existent que comme collectivité. Et alors que l'historiographie depuis vingt ans voit dans les congrégations féminines du XIX ${ }^{\mathrm{e}}$ siècle une voie possible de l'émancipation par l'accès à des postes de responsabilité ou nécessitant des compétences professionnelles ${ }^{89}$, Moulin lorsqu'il regarde les sœurs et qu'il décrit leur action les cantonne dans un rôle sexué traditionnel et anonyme. Elles sont, à l'en croire, au service des prêtres et de l'Église, rien de plus. Elles préparent les repas des missionnaires, elles décorent la chapelle. Certes

87. Julien Moulin à ses parents, Île-à-la-Crosse, 8 janvier 1864 .

88. Ainsi suite à un incendie en 1867 les deux prêtres, les deux frères et deux serviteurs se serrent quelque temps dans "vingt pieds carrés " 6 mai 1867 (cité plus haut).

89. LANGlois, C., Les Congrégations..., op. cit. Pour le cas nord-américain, voir CoBuRn, Carol K., Smith, M., Spirited Lives : How Nuns Shaped Catholic Culture and American Life, 1836-1920, Chapel Hill, University of North Carolina Press, 1999, 327 p. BRuno-Joffre, R., The Missionary Oblate Sisters : Vision and Mission, Montréal, McGill-Queen's Univerity Press, 2005, $220 \mathrm{p}$. 
elles font l'école aux enfants des Indiens, mais leur action dans ce cadre n'est guère commentée alors même qu'il est dit que « ces sœurs [...] sont très dévouées ${ }^{90}$ " aux deux prêtres du poste.

Le prêtre se pose donc en chef de sa petite communauté, isolé dans sa position supérieure. Par contre, à l'échelle diocésaine, il n'est qu'obéissance et respect face à son évêque. Mgrs Taché, Faraud, Grandin, et Clut sont omniprésents dans la correspondance de Moulin. L'admiration qu'il professe notamment pour Taché est manifeste dès son arrivée à SaintBoniface et ne se démentira plus. Entre les quatre prélats les relations ne furent pas toujours tendres. Le Nord-Ouest n'est pas exempt - le contraire serait étonnant - de tensions internes à l'Église ${ }^{91}$. Moulin n'en dit rien à ses parents. Soit qu'il ait tenu lui-même à se situer toujours du côté de la légitimité soit qu'il ne veuille ébruiter vers l'extérieur des querelles intestines, le silence qui enveloppe dans ses missives le fonctionnement interne de l'Église du Nord-Ouest ne laisse transparaître qu'une stricte hiérarchie dont on devine seulement qu'elle peut laisser place à des tensions, entre égaux - les récriminations contre un jeune prêtre ne parlant les langues - comme entre supérieurs et inférieurs.

Moulin est finalement plus disert sur ses relations avec l'extérieur : ce qui tient lieu d'autorité politique à savoir la Compagnie de la Baie d'Hudson, et les missionnaires protestants concurrents. Là les enjeux de pouvoir transparaissent davantage bien qu'encore avec discrétion. Moulin est averti dès son arrivée au Canada de l'importance de la Compagnie et de la nature des relations que l'Église entretient avec elle. Lorsqu'il relate à ses parents l'histoire de l'Île-à-la-Crosse avant qu'il ne s'y installe, il doit mentionner que sans l'asile que le poste de la Compagnie voulut bien donner aux missionnaires le travail de ces derniers aurait été très bref ${ }^{92}$. Lui-même ne cesse de l'expérimenter. Si les " traiteurs " peuvent par leur "mauvaise conduite " freiner l'œuvre de civilisation des "sauvages" en offrant de déplorables exemples ${ }^{93}$, les bourgeois de la Compagnie, eux, ne sont dignes que des plus grands éloges. Il se trouve qu'à l'Île-à-la-Crosse le responsable du poste est un des rares Canadien à avoir dépassé le stade du simple employé. Deschambault est donc catholique, et sa présence constitue un soutien indispensable au travail des missionnaires puisqu'il " fait tout ce qui est en lui pour la propagation de notre sainte foi ${ }^{94}$ ". " Nous sommes heureux d'avoir cette compagnie; car sans elle nous serions dans l'impossibilité de faire des missions ${ }^{95}$ ", en conclut Moulin. Mais l'inverse est vrai aussi. Car si l'infrastructure du commerce est indispensable au fonctionnement des missions, celles-ci sont vite perçues par la Compagnie comme également

90. 7 janvier 1869 (cité plus haut).

91. HuEl, R. J. A., Proclaming..., op. cit. et Archbishop..., op. cit.

92. 15 janvier 1860 (cité plus haut).

93. 28 mai 1862 (cité plus haut).

94. 12 juillet 1865 (cité plus haut).

95. 23 juillet 1863 (cité plus haut). 
utiles pour fixer et encadrer les populations indiennes. Aussi, que les missionnaires soient protestants ou catholiques importe peu aux bourgeois des postes de traite, en témoigne cette anecdote rapportée par Moulin :

" Je me suis trouvé à table avec un ministre protestant on lui a donné la première place d'honneur à moi la seconde le gouverneur a été placé après nous. Le ministre protestant a dit le bénédicité et le bourgeois en charge bien que protestant m'a demandé de dire les grâces ${ }^{96}$. "

Du fait de la politique et du commerce, Moulin doit donc en cet instant cohabiter avec un concurrent. Entre les anglicans et les catholiques il s'agit d'une sorte de course à la conversion des Indiens et aux faveurs de la Compagnie. Durant l'été 1860, alors que Moulin est à l'Île-à-la-Crosse, il apprend "l'arrivée [au Portage La Loge] d'un ministre protestant qui est venu au-devant de sa femme et qui pouvait faire beaucoup de mal parmi les sauvages ${ }^{97}$ ". Aussi part-il lui aussi en mission au même endroit pour contrer immédiatement l'ennemi. Pendant l'hiver 1863, au contraire, le catholique est vaincu, car le responsable britannique de Fort Carillon argue du manque de place pour refuser de le recevoir alors même qu'un missionnaire protestant semble venir s'y établir ${ }^{98}$. Il y a donc une réelle inimitié entre les représentants des deux christianismes, en même temps qu'ils peuvent se retrouver à la même table si la Compagnie les y invite car, malgré la concurrence, il existe entre eux un sentiment qui les unit, celui d'être " civilisé " et de remplir un devoir auprès des "sauvages ". Deux mondes semblent se faire face dans le Nord-Ouest pour Moulin, malgré les divisions parfois rudes au sein de chacun d'eux : les " civilisés " et les autres.

Ces autres, ce sont d'abord les Indiens, que Moulin, conformément à l'usage du temps, ne nomme que "sauvages ". Et, somme toute, il n'en dit rien à ses parents. Ou plutôt il ne décrit jamais leur manière de faire, de vivre. Tout juste doit-on deviner qu'ils sont nomades à quelques remarques éparses, et qu'il s'agit de Montagnais (Chipewyans) et de Cris. Ils sont en fait une sorte de masse anonyme d'où n'émergent que quelques cas propres à fournir des exempla édifiantes, destinées à prouver la toutepuissance divine et les succès du travail missionnaire. Un fils guérit grâce aux prières de ses parents ${ }^{99}$, une mourante se réjouit d'approcher Dieu au ciel ${ }^{100}$, un père cherche la consolation après la mort de son fils ${ }^{101}$, une " pauvre aveugle " veut faire bénir la terre où sa famille est enterrée ${ }^{102}$, un homme se résigne à la mort de ses enfants ${ }^{103}$. Il n'est donc question que d'apprentissage de la bonne mort, de la résignation et du bonheur de

96. 12 juillet 1865 (cité plus haut).

97. 30 juillet 1860 (cité plus haut).

98. 7 janvier 1863 (cité plus haut).

99. Ibidem.

100. 20 juillet 1863 (cité plus haut).

101. 29 mai 1866 (cité plus haut).

102. 7 janvier 1869 (cité plus haut).

103.5 août 1869 (cité plus haut). 
disparaître de cette terre pour rejoindre les cieux. Il s'agit d'une obsession personnelle de Moulin, qu'il s'applique aussi à lui-même, en affirmant lettre après lettre que le bonheur n'est pas de ce monde, où il faut se sacrifier pour espérer la félicité après la mort, dans l'union avec Jésus et les retrouvailles entre parents. On peut supposer que l'essentiel des instructions que Moulin donne aux Indiens tient du commentaire de ce thème, et ce n'est que par ce biais qu'il fait connaître ses ouailles à La Gouesnière :

" Je remercie Dieu de ce qu'il a bien voulu me choisir pour porter les lumières de la foi au milieu de ces peuples si malheureux, se dénués de tous les biens de la fortune de plus assis dans les ténèbres des ombres de la mort. Avant de connaître la religion ils étaient on pourrait dire sans Dieu sans espérance et malgré l'extrême misère ou ils se trouvaient, ils redoutaient la mort, mais maintenant il n'en est plus de même ils sont sinon contents de mourir du moins bien résignés ${ }^{104}$."

Moulin dit donc le succès, dix ans après son arrivée. Il est sans doute difficile de le suivre sur ce terrain tant la négation de l'être des Indiens ne pouvait que mener à l'inverse, c'est-à-dire un refus de la conversion ou une acceptation du catholicisme par l'intégration à la culture locale sans rejet de l'ancien, une sorte de "traduction" du christianisme ${ }^{105}$. L'attitude de Moulin, comme celle de ses collègues missionnaires, confine au refus de l'autre. Il n'est pas un prêtre ethnologue qui se pencherait sur les Chipewyan et les Cris pour les approcher. Il cherche à convaincre, et son pragmatisme se limite à l'adaptation matérielle aux conditions de la mission, sans aller jusqu'au contenu de l'enseignement religieux. Il ne voit pas les Indiens, mais de pauvres êtres vides de sens qu'il suffirait de remplir de son discours. Dans ce contexte il ne peut développer de véritables relations interpersonnelles, pas plus qu'il ne peut offrir une réflexion sur le destin du groupe.

Il en va différemment dès lors qu'il s'agit des Métis car même si leurs mœurs les apparentent pour les missionnaires - et pour beaucoup d'autres - aux " sauvages ", ils sont pour partie du côté de la " civilisation " du fait de leur ascendance canadienne et donc à la fois de leur catholicisme, même imparfait, et de leur langue, le français ${ }^{106}$. C'est par eux que Moulin a connu le Canada, dans la colonie de la Rivière Rouge : "Ce sont tous de

\section{Ibidem.}

105. Voir parmi d'autres Silverman, David J., Faith and Boundaries : Colonists, Christianity, and Community among the Wampanoag Indians of Martha's Vineyard, 1600-1807, New York, Cambridge University Press, 2005, 328 p.; GRUZINSKI, Serge, La colonisation de l'imaginaire, sociétés indigènes et occidentalisation dans le Mexique espagnol, XVI'-XVIII siècle, Paris, Gallimard, 1988.

106. ENs, Gerhard John, Homeland to Hinterland : The Changing Worlds of the Red River Metis in the Nineteenth Century, Toronto, University of Toronto Press, 1996, 268 p.; SAINTOnge, N., Saint-Laurent, Manitoba : Evolving Metis Identities, 1850-1914, Regina, Canadian Plains Research Center, 2004, 137 p. L'ouvrage de base reste : Giraud, Marcel, Le Métis canadien. Son rôle dans l'histoire des provinces de l'Ouest, Saint-Boniface, Éditions du Blé, 1984 [Paris, 1945], 2 vol., 1316 p. 
braves gens qui ont beaucoup de respect pour le prêtre ${ }^{107}$. " Et c'est par eux que s'achève la correspondance conservée dans les archives de La Gouesnière. En effet les trois dernières lettres du corpus, datées de 1877 et 1878 , sont isolées du reste d'un ensemble qui sans elles demeurerait cohérent et prendrait fin en 1871. Elles sont adressées par Moulin à son père devenu sourd (il mourra en 1880) alors que sa mère est décédée en 1872. Julien Moulin demeure alors de manière alternée auprès des Indiens et des Métis de la Saskatchewan. Sa vision des Indiens n'a pas évolué, mais il est davantage préoccupé par le sort des Métis. A priori ils semblent favorisés par Moulin. Mais le vice de leur mode de vie transparaît : leur nomadisme les fait basculer malgré tout du côté de la sauvagerie. Moulin semble les défendre, puisqu'il se range à leurs côtés, vit avec eux les chasses saisonnières comme les prêtres le faisaient depuis des décennies. Mais les Métis sont menacés : « Il serait bien à désirer que nos Métis quitâssent leur vie nomade et qu'ils se missent à cultiver car autrement les étrangers vont s'emparer des terres et ils vont se trouver contraints de s'éloigner d'avantage ${ }^{108}$. " Et Moulin regrette que ces étrangers ne soient pas plus nombreux. Ou plutôt il regrette que ne viennent pas en plus grand nombre des migrants français, " car [il est] convaincu que plusieurs pauvres de France y deviendraient bientôt riches ${ }^{109}$ ". Dix-neuf ans plus tôt, le même Moulin faisait part de ses premières impressions à ses parents : "Je suis persuadé que bon nombre d'ouvriers dans nos pays qui vivent avec beaucoup de peine seraient fort à leur aise ici. La terre ne coûte rien ou presque rien. Il suffit de faire des clôtures pour prendre possession ${ }^{110}$. "Le monde idéal de Moulin est demeuré le même: des communautés de paysans catholiques encadrées par leurs prêtres. Il aura sans cesse tenté de plier les Indiens et les Métis à sa logique de reproduction de son propre monde, fait d'enracinement dans le pays malouin, de solidarités familiales et de dogmes romains. L'épisode qui vaudra à Moulin de passer, avec modestie, à la postérité, se comprend alors : son refus de soutenir la révolte de Riel en 1885 en maintenant closes les portes de son église face aux rebelles est dans la continuité de son action depuis son arrivée au Canada. Il rêve avant tout d'un monde harmonieux, où les conflits se seraient réglés d'eux-mêmes, comme étouffés dans la vie immobile du village sous son autorité.

"À vous dire vrai depuis que je suis abandonné à la volonté sainte j'ai toujours été heureux partout où je suis allé ${ }^{111}$. " Julien Moulin aura tenu ce même discours durant les vingt ans que dure la correspondance conservée dans les archives de sa paroisse d'origine. Il n'aura eu de cesse de dire son bonheur dans le sacrifice et le don de soi, de dire que les épreuves n'étaient rien au regard de celles du Christ et que la joie de le retrou-

107. 22 janvier 1859 (cité plus haut).

108. Julien Moulin à son père, mission de Saint-Laurent, le 17 août 1877.

109. Julien Moulin à son père, mission de Saint-Julien Lac Vert, 27 mars 1878.

110. Le 22 janvier 1859 (cité plus haut).

111. Le 6 juin 1859 (cité plus haut). 
ver aux cieux valait bien tous les déchirements ici-bas. Nous touchons là une des limites de la source. Moulin utilise, jusqu'à répéter des formules bien apprises d'une lettre à l'autre, son acquis de prêtre et d'Oblat pour délivrer à ses parents, et se délivrer à soi-même sans doute, un message performatif, celui de l'assurance que son départ n'a pas mené à l'échec, ni sur le plan personnel - puisque le missionnaire se porte bien - ni sur le plan professionnel - puisque l'œuvre d'évangélisation avance. Il y a là une permanence de la morale catholique de l'humilité en même temps que le souci du migrant de produire un récit positif de son expérience. C'est dire qu'une correspondance missionnaire doit être analysée à l'aune de la double nature du scripteur, un migrant et un prêtre, et que les stratégies d'écriture en sont d'autant plus capitales à décrypter ${ }^{112}$. L'univers culturel du missionnaire ne peut être dévoilé qu'au prix de ce travail au plus près de l'homme de chair et de sang.

\section{RÉSUMÉ}

La figure du missionnaire, recouverte sous l'hagiographie ou récemment sous celle du récepteur du message chrétien, doit être réévaluée, ici à l'aune du cas de Julien Moulin, né à La Gouesnière en 1830 et parti comme Oblat en mission dans le Nord-Ouest canadien en 1858. Il s'agit de tenter d'analyser d'abord le milieu familial, social, religieux, qui l'a vu naître, et qui a pu le produire en tant que prêtre et missionnaire, avant de s'interroger sur les brisures provoquées chez lui par son expérience canadienne. S'interroger sur l'espace intérieur du missionnaire permet de mettre en relation le village breton, le séminaire et le geste migratoire dans un contexte extrême, et donc de placer ces prêtres au centre d'une histoire sociale et culturelle transatlantique.

\section{ABSTRACT}

Julien Moulin was born in La Gouesnière in 1830 and became a missionary in the Canadian North-West where he wanted to evangelize Natives and Metis from 1859 to his death in 1920. The archives of his own native parish contain many letters he sent to his family from 1854 to 1878. By this way we can observe Moulin's inner world. But this is not a simple and traditional biography. Here I try to make the history of a parish of Brittany in the first half of the 19 th century and the production of a priest; the history of the Canadian mission of the Oblates of Mary Immaculate; and particularly the history of the personal experience of the missionary. So this is above all the often forgotten atlantic cultural history of a missionary.

112. Sur la correspondance des missionnaires, ToulLELAN, Pierre-Yves, Missionnaires au quotidien à Tahiti : Les Picpusiens en Polynésie au XIX siècle, Leyde, Brill, 1995, 342 p., voir notamment le chapitre 5 . 